CUBO A Mathematical Journal

Vol.17, $N^{-} 01$, (29-40). March 2015

\title{
Maps preserving Fredholm or semi-Fredholm elements relative to some ideal
}

\author{
Mohadeseh Rostamani \\ Department of Pure Mathematics, \\ Ferdowsi University of Mashhad, \\ Mashhad, Iran. \\ mohadeseh.rostamani@gmail.com
}

\author{
SHIRIN HEJAZIAN \\ Department of Pure Mathematics, \\ Ferdowsi University of Mashhad, \\ Tusi Mathematical Research Group (TMRG), \\ Mashhad, Iran. \\ hejazian@um.ac.ir
}

\begin{abstract}
We consider the Calkin algebra $C_{R}(A)$ and the Fredholm theory in a Banach algebra $A$, relative to some fixed ideal $F$ of $A$. Our aim is to study linear maps between unital Banach algebras $A$ and $B$ which are surjective up to the inessential elements relative to $F$, and preserve Fredholm or semi-Fredholm elements in both directions or equivalently different relatively essential spectral sets such as essential spectrum, left or right essential spectrum, the boundary of essential spectrum or the full essential spectrum. We characterize such mappings when one of $C_{R}(A)$ or $C_{R}(B)$ is commutative and also investigate similar problems when $A$ is assumed to be a unital $C^{*}$-algebra of real rank zero and $B$ is an arbitrary Banach algebra.
\end{abstract}

\section{RESUMEN}

Consideramos el álgebra de Calkin $C_{R}(A)$ y la teoría de Fredholm en un álgebra de Banach $A$ relativa a algún ideal fijo $F$ de $A$. Nuestra meta es estudiar aplicaciones lineales entre álgebras de Banach unitales A y B las cuales son sobrejectivas salvo los elementos no esenciales relativos a $\mathrm{F}$ y preservan los elementos de Fredholm o semi-Fredholm en ambas direcciones o equivalentemente conjuntos espectrales esenciales relativos diferentes tales como el espectro esencial izquierdo o derecho, la frontera del espectro esencial o el espectro esencial completo. Caracterizamos dichas aplicaciones cuando uno de los $C_{R}(A)$ o $C_{R}(B)$ es conmutativo e investigamos problemas similares cuando $A$ se asume que es una $C^{*}$-álgebra unital de rango real cero y B es una álgebra de Banach cualquiera.

Keywords and Phrases: Linear preservers, Fredholm element, semi-Fredholm element, inessential ideal, relative Calkin algebra.

2010 AMS Mathematics Subject Classification: 47B49, 47A10. 


\section{Introduction}

Let $A$ and $B$ be algebras, and let $\mathcal{P}$ be a property. We say that a linear map $\theta: A \rightarrow B$

(i) preserves property $\mathcal{P}$, if for each $a \in A, \theta(a)$ has property $\mathcal{P}$ whenever a has property $\mathcal{P}$;

(ii) preserves property $\mathcal{P}$ in both directions, if for each $a \in A, \theta(a)$ has property $\mathcal{P}$ if and only if a has this property.

In operator theory, one of the most active research areas is linear preservers. The main problems in this subject concern with characterizing those linear maps that leave certain properties invariant. In the context of operator algebras, some of the most well known preserving problems deal with characterizing linear maps on the algebra of all bounded linear operators acting on a Banach space which preserve Fredholm, semi-Fredholm or generalized invertible operators. New contributions to the study of linear preserver problems in $\mathcal{L}(\mathrm{H})$, the algebra of all bounded linear operators on an infinite dimensional complex Hilbert space $\mathrm{H}$, have been recently made by several authors, see [3, 4, 5, 12, 13, 14]. In [11, Kim and Park investigated linear maps $\phi$ on a unital $C^{*}$-algebra $A$ of real rank zero that are surjective up to some fixed closed ideal $I$ and $\pi(a)$ is invertible in $A / I$ if and only if $\pi(\phi(A))$ is invertible in $A / I$, where $\pi: A \rightarrow A / I$ is the canonical quotient map. In this paper we consider Fredholm theory in a Banach algebra $A$ relative to some fixed ideal F, c.f. [16], and investigate linear preservers between Banach algebras.

In Section 2 we give some preliminaries concerning Fredholm theory in Banach algebras and the ideal of inessential elements relative to a fixed ideal. We will also consider the relative Calkin algebra of a Banach algebra with respect to some fixed ideal. Section 3 is devoted to investigating linear maps between two unital Banach algebras A and B which are surjective up to inessential elements and preserve any of the sets of Fredholm, left semi-Fredholm, right semi-Fredholm or semi-Fredholm elements in both directions (equivalently, preserves different spectral sets). We characterize such mappings whenever one of the relative Calkin algebras in A or B is assumed to be commutative. In Section 4 we consider similar linear preservers between unital $\mathrm{C}^{*}$-algebras or from a unital $\mathrm{C}^{*}$-algebra of real rank zero to an arbitrary unital Banach algebra. In each case we show that a linear map which is surjective up to inessential ideal and preserves a certain essential spectral set, induces a continuous Jordan isomorphism between the corresponding relative Calkin algebras.

\section{Preliminaries}

Throughout this section $A$ is a unital Banach algebra with unit element 1 and $F$ is a fixed two sided ideal of $A$. The elements of $F$ are called finite elements of $A$. Set

$$
I(A)=k(h(F)):=\bigcap\left\{P \in \Pi_{A} ; F \subseteq P\right\},
$$

where $\Pi_{A}$ denotes the set of all primitive ideals of $A$. By [15, Theorem 4.3],

$$
I(A)=\{a \in A ; a+\bar{F} \in \operatorname{rad}(A / \bar{F})\}
$$


Clearly $I(A)$ is a closed two sided ideal of $A$ containing $F$. We call $I(A)$ the inessential ideal of $A$ (relative to $F$ ), and the elements of $I(A)$ are called the inessential elements relative to $F$.

Definition 2.1. An element $\mathrm{a} \in \mathrm{A}$ is said to be left (resp. right) semi-Fredholm (relative to $\mathrm{F}$ ), if it is left (resp. right) invertible modulo $\mathrm{F}$, and $\mathrm{a}$ is called Fredholm if it is invertible modulo $\mathrm{F}$. An element $\mathrm{a} \in \mathrm{A}$ is a semi-Fredholm or an Atkinson element if it is either left or right semi-Fredholm.

We denote by $\Phi_{l}(A)$ (resp. $\Phi_{\mathrm{r}}(A)$ ) the set of all left (resp. right) semi-Fredholm elements of A. Also we denote by $\Phi(A):=\Phi_{l}(A) \cap \Phi_{r}(A)$ and $\Psi(A):=\Phi_{l}(A) \cup \Phi_{r}(A)$ the sets of Fredholm and semi-Fredholm elements of $A$, respectively.

By [2, BA.2.4] an element $a \in A$ is left (resp. right) invertible modulo $F$ if and only if it is left (resp. right) invertible modulo $I(A)$.

Since $I(A)$ is a closed ideal it is easy to see that $\Phi(A), \Phi_{l}(A)$ and $\Phi_{r}(A)$ are open multiplicative semigroups of $A$ and adding $I(A)$ to each of these classes leaves it stable. For more about Fredholm theory in Banach algebras see [2, 15, 16].

Definition 2.2. The quotient algebra $A / I(A)$, denoted by $C_{R}(A)$, is called the relative Calkin algebra of $\mathrm{A}$ with respect to $\mathrm{F}$.

The relative Calkin algebra of $A$ is semisimple; see [16, Theorem 3.2 (vi)].

We recall that if $A$ has minimal left ideals the smallest left ideal containing all of them is called the left socle of $\mathrm{A}$. The right socle is similarly defined in terms of right ideals. If $\mathrm{A}$ has both minimal left and minimal right ideals, and if the left socle coincides with the right socle, it is called the socle of $A$ denoted by $\operatorname{soc}(A)$. In this case we say for brevity that $\operatorname{soc}(A)$ exists. If $A$ has no minimal left or minimal right ideals we put $\operatorname{soc}(A)=\{0\}$. Clearly the socle, if it exists, is a non-zero ideal of $\mathrm{A}$. If $\mathrm{A}$ is a semiprime algebra which has minimal left ideals, then $\operatorname{soc}(\mathrm{A})$ exists (see [2, BA.3.3]).

When $A$ is a semisimple Banach algebra, as a special case in Definition 2.2. we may assume $F$ to be the socle of $A$. In this case the relative Calkin algebra coincides with the generalized Calkin algebra as in 4 .

For $x, y \in A$ let $x \circ y=x+y-x y$ denote the quasi product of $x$ and $y$. Set

$$
\mathrm{Q}(\mathrm{A}):=\{x \in A \text {; there exist } u, v \in A \text { such that } u \circ x, x \circ v \in F\} .
$$

By [16. Theorem 3.2 (viii)], $I(A)$ is the largest ideal of $A$ contained in $Q(A)$.

Lemma 2.3. Let $\mathrm{A}$ be a unital Banach algebra. Then

$$
\begin{aligned}
I(A) & =\{x \in A ; u+x \in \Phi(A) \text { for all } u \in \Phi(A)\} \\
& =\left\{x \in A ; u+x \in \Phi_{l}(A) \text { for all } u \in \Phi_{l}(A)\right\} \\
& =\left\{x \in A ; u+x \in \Phi_{r}(A) \text { for all } u \in \Phi_{r}(A)\right\} \\
& =\{x \in A ; u+x \in \Psi(A) \text { for all } u \in \Psi(A)\} .
\end{aligned}
$$


Proof. The first equality is [16, Theorem 3.5]. We prove the second equality; the others are proved similarly. Let

$$
J=\left\{x \in A ; u+x \in \Phi_{l}(A) \text { for all } u \in \Phi_{l}(A)\right\} .
$$

Clearly $I(A) \subseteq J$. A modification of the proof of [16, Theorem 3.5] shows that $J$ is an ideal of A. We show that $\mathrm{J} \subseteq \mathrm{Q}(A)$. Let $x \in \mathrm{J}$, then $1-x \in \Phi_{l}(A)$. Thus there exists $y \in A$ such that $(1-y)(1-x)-1 \in F$. Since $F \subseteq J$ we have $y \in J$. Therefore there exists $z \in A$ such that $(1-z)(1-y)-1 \in F$. This shows that $1-y+F$ is both left and right invertible in $A / F$ with right inverse $1-x+F$. It follows that $1-x+F$ is invertible in $A / F$ and so $x \in Q(A)$, therefore $J \subseteq Q(A)$. Now [16, Theorem 3.2 (viii)] implies that $J=I(A)$.

In [4, Lemma 3.2] a different method has been applied to prove the same equalities as in Lemma 2.3 when $A$ is assumed to be semisimple and $F$ is assumed to be the socle of $A$. However, it should be emphasized that semisimplicity has been used just to guarantee the existence of the socle.

For any $a \in A$

$$
\begin{aligned}
\sigma(a) & :=\{\lambda \in \mathbb{C}: a-\lambda 1 \text { is not invertible }\} \\
\sigma_{l}(a) & :=\{\lambda \in \mathbb{C}: a-\lambda 1 \text { is not left invertible }\} \\
\sigma_{\mathrm{r}}(a) & :=\{\lambda \in \mathbb{C}: a-\lambda 1 \text { is not right invertible }\}
\end{aligned}
$$

denote the spectrum, the left spectrum and the right spectrum of a, respectively. Also $\partial \sigma(a)$ and $r(a)$ are the boundary of the spectrum and the spectral radius of $a$, respectively. We recall that for every compact set $\mathrm{K} \subseteq \mathbb{C}$, the polynomial convex hull $\eta \mathrm{K}$, is defined by

$$
\eta \mathrm{K}=\left\{z \in \mathbb{C}:|p(z)| \leq \sup _{\xi \in \mathrm{K}}|p(\xi)|, \text { for each polynomial } p\right\} .
$$

For an element $a$ in a Banach algebra, the polynomial convex hull $\eta \sigma(x)$ of $\sigma(x)$ is called the full spectrum of $a$.

Let $\pi_{A}: A \rightarrow C_{R}(A)$ denote the canonical quotient map. For $a \in A, \sigma_{e}(a):=\sigma\left(\pi_{A}(a)\right)$, $\sigma_{l e}(a):=\sigma_{l}\left(\pi_{A}(a)\right), \sigma_{r e}(a):=\sigma_{r}\left(\pi_{A}(a)\right), \eta \sigma_{e}(a):=\eta \sigma\left(\pi_{A}(a)\right)$ and $r_{e}(a):=r\left(\pi_{A}(a)\right)$ are called, respectively, the essential spectrum, the left essential spectrum, the right essential spectrum, the full essential spectrum and the essential spectral radius of a relative to $F$.

Let $\Lambda($.$) denote any of the spectral sets \sigma_{e}(),. \sigma_{l e}(),. \sigma_{\text {re }}(),. \sigma_{l e}(.) \cap \sigma_{\mathrm{re}}(),. \partial \sigma_{e}($.$) and \eta \sigma_{e}($.$) .$ A linear map $\theta: A \rightarrow B$ is said to be $\Lambda($.)-preserving if $\Lambda(\theta(x))=\Lambda(x)$ for all $x \in A$, and $\theta$ is called essentially spectrally bounded if there exists a positive constant $M$ such that $r_{e}(\theta(x)) \leq M r_{e}(x)$ for all $x \in A$. If $r_{e}(\theta(x))=r_{e}(x)$ for all $x \in A, \theta$ is called an essential spectral isometry.

Throughout this paper we will always suppose the following.

Assumption A. We assume that $\mathrm{A}$ and $\mathrm{B}$ are unital Banach algebras and denote by 1 the unit element both in $\mathrm{A}$ and $\mathrm{B}$. We consider arbitrary fixed ideals $\mathrm{F}$ and $\mathrm{F}^{\prime}$ in $\mathrm{A}$ and $\mathrm{B}$, respectively. The inessential ideals in $\mathrm{A}$ and $\mathrm{B}$ relative to $\mathrm{F}$ and $\mathrm{F}^{\prime}$ are denoted by $\mathrm{I}(\mathrm{A})$ and $\mathrm{I}(\mathrm{B})$, respectively. $\mathrm{C}_{\mathrm{R}}($. 
denotes the relative Calkin algebra in the corresponding Banach algebra. Note that when we use the terms Fredholm, left semi-Fredholm, right semi-Fredholm or semi-Fredholm, we always mean relative to the specific fixed ideal considered in the corresponding algebra.

For a linear map $\theta: A \rightarrow B, N(\theta)$ will denote the null space of $\theta$. If $\theta(1)=1, \theta$ is called unital. We say that $\theta$ is surjective up to inessential ideal if $B=\theta(A)+I(B)$. If $\theta\left(a^{2}\right)=\theta(a)^{2}$ for all $a \in A$ (equivalently, $\theta(a b+b a)=\theta(a) \theta(b)+\theta(b) \theta(a)$ for all $a, b \in A$ ), then $\theta$ is said to be $a$ Jordan homomorphism. A Jordan isomorphism is a bijective Jordan homomorphism.

\section{$3 \quad$ Fredholm and semi-Fredholm preservers}

We consider Assumption A and begin with the following lemma. Note that there is no assumption of semisimplicity neither for $A$ nor for B. The proof of the following lemma is a modification of a part of the proof of [3, Theorem 1.1]. We give the proof for the sake of convenience.

Lemma 3.1. If the linear map $\theta: \mathrm{A} \rightarrow \mathrm{B}$ is surjective up to the inessential ideal and essentially spectrally bounded then $\theta(\mathrm{I}(\mathrm{A})) \subseteq \mathrm{I}(\mathrm{B})$.

Proof. Assume that there is a positive constant $M$ such that $r_{e}(\theta(a)) \leq M_{e}(a)$ for all $a \in A$. Take $u \in I(A)$ and let $b \in B$ be an arbitrary element. Since $\theta$ is surjective up to the inessential ideal, there exist $x \in A$ and $y \in I(B)$ such that $b=\theta(x)+y$. For every $\lambda \in \mathbb{C}$, we have

$$
\begin{aligned}
r\left(\lambda \pi_{\mathrm{B}}(\theta(u))+\pi_{\mathrm{B}}(\mathrm{b})\right) & =r\left(\pi_{\mathrm{B}}(\lambda \theta(u)+b)\right)=r_{e}(\lambda \theta(u)+b) \\
& =r_{e}(\theta(\lambda u+x)+y)=r_{e}(\theta(\lambda u+x)) \\
& \leq \operatorname{Mr}_{e}(\lambda u+x)=\operatorname{Mr}_{e}(x) .
\end{aligned}
$$

The function $\lambda \mapsto r\left(\lambda \pi_{\mathrm{B}}(\theta(\mathfrak{u}))+\pi_{\mathrm{B}}(\mathrm{b})\right)$ is subharmonic on $\mathbb{C}$, and so by Liouville's Theorem $r\left(\pi_{B}(\theta(u))+\pi_{B}(b)\right)=r\left(\pi_{B}(b)\right)$. Zemanek's characterization of the radical (see 11, Theorem 5.3.1]), implies that $\pi_{B}(\theta(u)) \in \operatorname{rad}\left(C_{R}(B)\right)=\{0\}$ and thus $\theta(u) \in I(B)$.

Lemma 3.2. Let the linear map $\theta: \mathrm{A} \rightarrow \mathrm{B}$ be surjective up to the inessential ideal. If $\theta$ preserves any of the sets of Fredholm, left semi-Fredholm, right semi-Fredholm or semi-Fredholm elements in both directions, then

(i) $\theta(\mathrm{I}(\mathrm{A})) \subseteq \mathrm{I}(\mathrm{B})$;

(ii) $N(\theta) \subseteq I(A)$.

Proof. (i) If $\theta(1)=1(\bmod I(B))$, then $r_{e}(\theta(a))=r_{e}(a)$ for all $a \in A$ and the result follows by Lemma 3.1 Otherwise, let $S($.$) denote any of the sets of Fredholm, left semi-Fredholm, right semi-$ Fredholm or semi-Fredholm elements in the corresponding Banach algebra. Let $x \in I(A)$. Then we show that $\theta(x) \in I(B)$. Suppose $u \in S(B)$. Since $\theta$ is surjective up to the inessential ideal $I(B)$, 
there exist $u^{\prime} \in A$ and $x^{\prime} \in I(B)$ such that $u=\theta\left(u^{\prime}\right)+x^{\prime}$. It follows that $\theta\left(u^{\prime}\right)=u-x^{\prime} \in S(B)$ and so $u^{\prime} \in S(A)$. Now $u+\theta(x)=\theta\left(u^{\prime}\right)+x^{\prime}+\theta(x)=\theta\left(u^{\prime}+x\right)+x^{\prime} \in S(B)$. Thus, for every $u \in S(B)$, $u+\theta(x) \in S(B)$. Lemma 2.3 implies that $\theta(x) \in I(B)$, which establishes that $\theta(I(A)) \subseteq I(B)$.

(ii) Let $S($.) be as above. If $x \in N(\theta)$ and $u \in S(A)$, then $\theta(x+\mathfrak{u})=\theta(u) \in S(B)$. Thus, for all $u \in S(A), x+u \in S(A)$ and by Lemma 2.3, $x \in I(A)$.

Remark 3.3. By [1, Corollary 5.2.3] a semisimple Banach algebra $\mathrm{A}$ is commutative if and only if the spectral radius is subadditive on $A$, that is, there exists $M>0$ such that $r(x+y) \leq$ $\mathrm{M}(\mathrm{r}(\mathrm{x})+\mathrm{r}(\mathrm{y}))(\mathrm{x}, \mathrm{y} \in \mathrm{A})$. Now, let $\mathrm{A}$ and $\mathrm{B}$ be semisimple Banach algebras and let $\theta: \mathrm{A} \rightarrow \mathrm{B}$ be a surjective linear map which preserves spectral radius. It is easy to see that commutativity of either of A or B, implies commutativity of the other one.

Theorem 3.4. Let at least one of $\mathrm{C}_{\mathrm{R}}(\mathrm{A})$ or $\mathrm{C}_{\mathrm{R}}(\mathrm{B})$ be commutative. Suppose that $\theta: \mathrm{A} \rightarrow \mathrm{B}$ is a linear map which is surjective up to the inessential ideal. Then the following assertions are equivalent.

(i) $\theta$ preserves the set of Fredholm elements in both directions;

(ii) $\theta(\mathrm{I}(\mathrm{A})) \subseteq \mathrm{I}(\mathrm{B})$ and the induced map $\widehat{\theta}: \mathrm{C}_{\mathrm{R}}(\mathrm{A}) \rightarrow \mathrm{C}_{\mathrm{R}}(\mathrm{B}), \widehat{\theta} \circ \pi_{\mathrm{A}}=\pi_{\mathrm{B}} \circ \theta$, is a continuous isomorphism multiplied by an invertible element in $\mathrm{C}_{\mathrm{R}}(\mathrm{B})$.

Proof. $(\mathfrak{i}) \Rightarrow$ (ii). By Lemma 3.2, $\theta(I(A)) \subseteq I(B))$; thus $\theta$ induces a linear map $\widehat{\theta}: C_{R}(A) \rightarrow C_{R}(B)$ such that $\widehat{\theta} \circ \pi_{A}=\pi_{B} \circ \theta$. Clearly $\widehat{\theta}$ is surjective. Since $\theta(1)$ is a Fredholm element, $\pi_{B}(\theta(1))$ is invertible in $C_{R}(B)$. Take $s \in B$ such that $\pi_{B}(s)=\pi_{B}(\theta(1))^{-1}$ in $C_{R}(B)$. Consider

$$
\psi:=\mathrm{L}_{\pi_{\mathrm{B}}(s)} \circ \widehat{\theta}: \mathrm{C}_{\mathrm{R}}(\mathrm{A}) \rightarrow \mathrm{C}_{\mathrm{R}}(\mathrm{B}),
$$

where the linear mapping $L_{\pi_{B}(s)}$ is the left multiplication by $\pi_{B}(s)$ in $C_{R}(B)$. Then $\psi\left(\pi_{A}(x)\right)=$ $\pi_{B}(s \theta(x))$ for all $x \in A$. Now it is easy to see that $\psi$ is surjective, $\psi\left(\pi_{A}(1)\right)=\pi_{B}(1)$ and

$$
\begin{aligned}
\pi_{A}(x) \in \operatorname{Inv}\left(C_{R}(A)\right) \Longleftrightarrow x \in \Phi(A) & \Longleftrightarrow \theta(x) \in \Phi(B) \\
& \Longleftrightarrow \pi_{B}(\theta(x)) \in \operatorname{Inv}\left(C_{R}(B)\right) \\
& \Longleftrightarrow \pi_{B}(s) \pi_{B}(\theta(x)) \in \operatorname{Inv}\left(C_{R}(B)\right) \\
& \Longleftrightarrow \psi\left(\pi_{A}(x)\right) \in \operatorname{Inv}\left(C_{R}(B)\right) .
\end{aligned}
$$

Thus $\psi$ is unital and preserves the set of invertible elements in both directions and so it preserves the spectrum. The Banach algebras $C_{R}(A)$ and $C_{R}(B)$ are semisimple, so by Remark 3.3 both $C_{R}(A)$ and $C_{R}(B)$ are commutative. It follows from [1, Theorem 4.1.17] that $\psi$ is an isomorphism which is continuous by semisimplicity of $C_{R}(B)$. The assertion (ii) $\Rightarrow(i)$ is obvious.

We recall that a $C^{*}$-algebra $A$ is said to be of real rank zero, if the set of all invertible selfadjoint elements of $A$ is dense in the set of selfadjoint elements. Suppose that $A$ is a $C^{*}$-algebra of real rank zero and $B$ is a semisimple Banach algebra. Let $I(A)$ and $I(B)$ denote the inessential ideals 
relative to $\operatorname{soc}(A)$ and $\operatorname{soc}(B)$, respectively. It is proved in [4, Corollary 3.5] that if $\theta: A \rightarrow B$ is a linear map which is surjective up to $I(B)$ and if $\theta$ preserves any of the sets $\Phi_{l}(A), \Phi_{r}(A)$ or $\Psi(A)$ in both directions, then $\theta(\mathrm{I}(A)) \subseteq \mathrm{I}(\mathrm{B})$ and the induced map $\widehat{\theta}: \mathrm{C}(\mathrm{A}) \rightarrow \mathrm{C}(\mathrm{B})$ is a continuous Jordan isomorphism multiplied by an invertible element in $C(B)$. Here we prove a similar result in a different setting.

Proposition 3.5. Let A, B satisfy Assumption A. Suppose that $\theta: A \rightarrow B$ is a linear map which is surjective up to the inessential ideal. If $\theta$ preserves any of the sets of left semi-Fredholm, right semiFredholm or semi-Fredholm elements in both directions, then $\theta(\mathrm{I}(\mathrm{A})) \subseteq \mathrm{I}(\mathrm{B})$ and $\theta$ preserves the set of Fredholm elements in both directions. Moreover, if one of $C_{R}(A)$ or $C_{R}(B)$ is commutative, then the induced map $\widehat{\theta}: \mathrm{C}_{\mathrm{R}}(\mathrm{A}) \rightarrow \mathrm{C}_{\mathrm{R}}(\mathrm{B})$ is a continuous isomorphism multiplied by an invertible element in $\mathrm{C}_{\mathrm{R}}(\mathrm{B})$.

Proof. By assumption and Lemma 3.2, $\widehat{\theta}: C_{R}(A) \rightarrow C_{R}(B)$ is well defined. Since $C_{R}(A), C_{R}(B)$ are semisimple, [4, Theorem 2.2] implies that $\widehat{\theta}$ preserves invertibility in both directions. Therefore $\theta$ preserves the set of Fredholm elements in both directions. The last assertion follows from Theorem 3.4

We will need the following theorem in the sequel. We recall that a linear map $\theta: A \rightarrow B$ preserves idempotents if for each idempotent $e$ in $A, \theta(e)$ is an idempotent in $B$.

Theorem 3.6. [10, Corollary 2.3] Let $\mathrm{A}$ and $\mathrm{B}$ be two semisimple Banach algebras and let $\Lambda($. denote any of the spectral sets $\sigma(),. \sigma_{l}(),. \sigma_{\mathrm{r}}(),. \sigma_{l}(.) \cap \sigma_{\mathrm{r}}(),. \partial \sigma($.$) and \eta \sigma($.$) . Suppose that \theta: A \rightarrow B$ is a surjective linear map. If $\Lambda(\theta(x))=\Lambda(x)$ for every $x \in A$, then $\theta$ is a bijective linear map preserving idempotents.

In the above theorem, since $\Lambda(\theta(x))=\Lambda(x)$ for one of the spectral sets, we have $\theta(1)=1$.

Remark 3.7. Let $\mathrm{K} \subseteq \mathbb{C}$ be compact. It is well known that $\partial \eta(\mathrm{K})$ is the boundary of the unbounded component of $\mathbb{C} \backslash \mathrm{K}$ which is called the outer boundary of $\mathrm{K}$. Thus $\max \{|z|: z \in \mathrm{K}\}=\max \{|z|: z \in$ $\partial \eta(K)\}$.

Theorem 3.8. Let $\mathrm{C}_{\mathrm{R}}(\mathrm{A})$ or $\mathrm{C}_{\mathrm{R}}(\mathrm{B})$ be commutative. Suppose that $\theta: \mathrm{A} \rightarrow \mathrm{B}$ is a linear map which is surjective up to the inessential ideal. The following assertions are equivalent.

(i) $\theta$ preserves the set of Fredholm elements in both directions and $\theta(1)=1(\bmod \mathrm{I}(\mathrm{B}))$.

(ii) $\theta(\mathrm{I}(\mathrm{A})) \subseteq \mathrm{I}(\mathrm{B})$ and the induced map $\widehat{\theta}: \mathrm{C}_{\mathrm{R}}(\mathrm{A}) \rightarrow \mathrm{C}_{\mathrm{R}}(\mathrm{B}), \widehat{\theta} \circ \pi_{\mathrm{A}}=\pi_{\mathrm{B}} \circ \theta$, is a continuous unital isomorphism.

(iii) $\theta$ is $\sigma_{e}$-preserving;

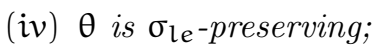

(v) $\theta$ is $\sigma_{\mathrm{re}-p r e s e r v i n g ;}$ 
(vi) $\theta$ is $\left(\sigma_{l e} \cap \sigma_{\text {re }}\right)$-preserving;

(vii) $\theta$ preserves the set of left semi-Fredholm elements in both directions and $\theta(1)=1(\bmod \mathrm{I}(\mathrm{B}))$.

(viii) $\theta$ preserves the set of right semi-Fredholm elements in both directions and $\theta(1)=1(\bmod \mathrm{I}(\mathrm{B}))$.

(ix) $\theta$ preserves the set of semi-Fredholm elements in both directions and $\theta(1)=1(\bmod \mathrm{I}(\mathrm{B}))$.

(x) $\theta$ is $\partial \sigma_{e}$-preserving;

(xi) $\theta$ is $\eta \sigma_{e}$-preserving.

(xii) $\theta$ is an essential spectral isometry and $\theta(1)=1(\bmod \mathrm{I}(\mathrm{B}))$.

Proof. $(i) \Rightarrow(i i)$. Since $\theta(1)=1(\bmod I(B))$, we have the result from Theorem 3.4 .

(ii) $\Rightarrow$ (iii). For every $x \in A$, we have

$$
\sigma_{e}(\theta(x))=\sigma\left(\pi_{B}(\theta(x))\right)=\sigma\left(\widehat{\theta}\left(\pi_{\mathrm{A}}(x)\right)\right)=\sigma\left(\pi_{\mathrm{A}}(x)\right)=\sigma_{e}(x)
$$

and the result holds.

$(\mathfrak{i i i}) \Rightarrow(\mathfrak{i})$. Since $\theta$ is $\sigma_{e}$-preserving, it preserves Fredholm elements in both directions. Lemma 3.2 implies that $\theta(I(A)) \subseteq I(B)$ and by the hypothesis the induced map $\widehat{\theta}: C_{R}(A) \rightarrow C_{R}(B)$ is spectrum preserving. It follows from Theorem 3.6 that $\widehat{\theta}$ is unital, that is $\theta(1)-1 \in I(B)$.

It is easy to see that (ii) implies all other assertions.

$(i v) \Rightarrow(v i i)$. If $\theta$ is $\sigma_{l e}$-preserving then $\theta$ preserves the set of left semi-Fredholm elements in both directions. By Lemma 3.2, $\widehat{\theta}$ is well defined and by the hypothesis it preserves the left spectrum. So by Theorem 3.6, $\widehat{\theta}$ is unital. The inverse implication is obvious. The assertions $(v) \Longleftrightarrow(v i i i)$, $(v i) \Longleftrightarrow(i x)$ are proved similarly.

(vii) $\Rightarrow$ (i). If $\theta$ preserves the set of left semi-Fredholm elements in both directions then by Proposition 3.5, $\theta$ preserves the set of Fredholm elements in both directions.

The same reasoning gives (viii) $\Rightarrow(\mathfrak{i})$ and $(\mathfrak{i x}) \Rightarrow(\mathfrak{i})$.

If each of assertions $(x),(x i)$ and (xii) holds then by Lemma 3.1, $\widehat{\theta}$ is well defined and from Theorem [3.6] $\widehat{\theta}$ is bijective, unital and also a spectral isometry. Thus both $C_{R}(A)$ and $C_{R}(B)$ are commutative. Now from [1, Theorem 4.1.17], $\widehat{\theta}$ is an isomorphism which is continuous by semisimplicity of $\mathrm{C}(\mathrm{B})$ and (ii) follows.

\section{Essential spectral preservers on $\mathrm{C}^{*}$-algebras}

Let $A$ be a Banach algebra. We recall that an element $a \in A$ is called regular if there exists $b \in A$ such that $a=a b a$ and $b=b a b ; b$ is said to be a generalized inverse for $a$. Note that if $a$ and $b$ in A satisfy $a=a b a$, then $a$ is regular and $b^{\prime}=b a b$ is a generalized inverse for $a$. This shows, in particular, that the generalized inverse of a regular element is not unique. For an element $a$ in a Banach algebra $A$, the regular set of a denoted by $\operatorname{reg}(a)$ is the set of all $\lambda \in \mathbb{C}$ such that there 
exists a neighborhood $U_{\lambda}$ of $\lambda$, and an analytic function $f: U_{\lambda} \rightarrow A$, such that $f(\mu)$ is a generalized inverse of $a-\mu 1$ for all $\mu \in U_{\lambda}$. The generalized spectrum (also called Saphar spectrum) of $a$ is given by $\sigma_{g}(a):=\mathbb{C} \backslash \operatorname{reg}(a)$. The conorm or the reduced minimum modulus of $a \in A$ is given by

$$
\gamma(a):= \begin{cases}\inf \left\{\|a x\|: \operatorname{dist}\left(x, \operatorname{ker}\left(L_{a}\right)\right) \geq 1\right\}, & \text { if } a \neq 0 \\ \infty, & \text { if } a=0,\end{cases}
$$

where $L_{a}$ denotes the operator $x \mapsto a x$ on $A$. If $F$ is a fixed ideal in $A$ then the essential conorm and the generalized essential spectrum of a relative to $F$ is given by $\gamma_{e}(a):=\gamma\left(\pi_{A}(a)\right)$ and $\sigma_{g e}(a):=\sigma_{g}\left(\pi_{A}(a)\right)$, respectively. Here $\pi_{A}$ denotes the canonical quotient map from $A$ onto the relative Calkin algebra $C_{R}(A)$. It is easy to see that if $A$ is a $C^{*}$-algebra then $\sigma_{g e}(a)=$ $\left\{\lambda \in \mathbb{C}: \lim _{\mu \rightarrow \lambda} \gamma_{e}(a-\mu)=0\right\}$, and that $\partial \sigma_{e}(a) \subseteq \sigma_{g e}(a) \subseteq \sigma_{e}(a)$. It is clear from the definition of $\gamma($.$) that, if \theta$ preserves essential conorm that is, $\gamma_{e}(\theta(a))=\gamma_{e}(a)$ for all $a \in A$, and $\theta(1)=1(\bmod I(B))$, then $\sigma_{g e}(\theta(a))=\sigma_{g e}(a)$ for all $a \in A$.

We recall that a unital $C^{*}$-algebra $A$ is said to be finite if $a^{*} a=1$ implies that $a a^{*}=1$. It is easy to see that in a finite $\mathrm{C}^{*}$-algebra every one-sided invertible element is invertible. $\mathrm{C}^{*}$-algebras with finite traces and those with dense subset of invertible elements are examples of finite $\mathrm{C}^{*}$ algebras. The quotient of a finite $C^{*}$ algebra $A$ need not be finite. A unital $C^{*}$ algebra $A$ is said to be residually finite if every quotient of $A$ is finite; [6, V.2.1.3].

Note that, in this section $A$ and $B$ as Banach algebras, satisfy Assumption A.

Theorem 4.1. Let $\mathrm{A}$ and $\mathrm{B}$ be unital $\mathrm{C}^{*}$-algebras and let $\theta: \mathrm{A} \rightarrow \mathrm{B}$ be a $*$-preserving linear map which is surjective up to the inessential ideal. Then in the following assertions, $(\mathfrak{i})-(\mathfrak{i} v)$ are equivalent and $(v)-(v i i)$ imply (ii). Moreover, if $\mathrm{A}$ and $\mathrm{B}$ are residually finite $\mathrm{C}^{*}$-algebras, then all of the following conditions are equivalent.

(i) $\theta$ is $\sigma_{e}$-preserving;

(ii) $\theta(\mathrm{I}(\mathrm{A})) \subseteq \mathrm{I}(\mathrm{B})$ and the induced map $\widehat{\theta}: \mathrm{C}_{\mathrm{R}}(\mathrm{A}) \rightarrow \mathrm{C}_{\mathrm{R}}(\mathrm{B})$, is an isometric Jordan isomorphism;

(iii) $\theta$ preserves Fredholm elements in both directions and $\theta(1)=1(\bmod \mathrm{I}(\mathrm{B}))$;

(iv) $\gamma_{e}(\theta(a))=\gamma_{e}(a)$ for all $\mathrm{a} \in A$ and $\theta(1)=1(\bmod \mathrm{I}(\mathrm{B}))$.

(v) $\theta$ is $\sigma_{l e}$-preserving;

(vi) $\theta$ is $\sigma_{\text {re }}$-preserving;

(vii) $\theta$ is $\left(\sigma_{l e} \cap \sigma_{\text {re }}\right)$-preserving.

Proof. $(\mathfrak{i}) \Rightarrow$ (iii). Since $\theta$ is $\sigma_{e}$-preserving, it preserves Fredholm elements in both directions and it follows from Lemma 3.2 that $\widehat{\theta}: C_{R}(A) \rightarrow C_{R}(B)$ is well defined and is unital by Theorem 3.6. (iii) $\Rightarrow$ (ii). It follows from Lemma 3.2 that $\widehat{\theta}$ is well defined and by the assumption it is unital 
and preserves invertibility in both directions. It is easy to see that $\widehat{\theta}$ is $*$-preserving. Therefore, $\widehat{\theta}$ is a Jordan isomorphism by [9] and is an isometry by [8, Lemma 4.3]. (ii) $\Rightarrow(\mathfrak{i})$ is obvious.

$(\mathfrak{i} v) \Rightarrow(\mathfrak{i i})$. If $(\mathfrak{i} v)$ holds then $\sigma_{g e}(\theta(a))=\sigma_{g e}(a)$ for all $a \in A$ and thus $\theta$ is an essential spectral isometry and so $\widehat{\theta}$ is well defined by Lemma 3.1. Moreover, $\widehat{\theta}$ is injective and preserves the conorm. Thus by [8, Theorem 3.1], $\widehat{\theta}$ is an isometric Jordan isomorphism.

(ii) $\Rightarrow(\mathfrak{i v})$ follows easily.

Now suppose that $(v)$ holds. Then $\theta$ preserves the set of left semi-Fredholm elements in both directions. Lemma 3.2 implies that $\widehat{\theta}$ is well defined. By Theorem 3.6 $\widehat{\theta}$ is unital and bijective. $\widehat{\theta}$ preserves left invertibility in both directions and so preserves invertibility in both directions by 44, Theorem 2.2]. Therefore $\widehat{\theta}\left(\operatorname{Inv}\left(C_{R}(A)\right)\right)=\operatorname{Inv}\left(C_{R}(B)\right)$. It is easy to see that $\widehat{\theta}$ is $*$-preserving. Therefore, $\widehat{\theta}$ is a Jordan isomorphism by [9] and is an isometry by [8, Lemma 4.3]. (vi) $\Rightarrow(\mathfrak{i i})$ and (vii) $\Rightarrow$ (ii) are proved in a similar way.

Finally, if $A$ and $B$ are residually finite $C^{*}$-algebras, then $C_{R}(A)$ and $C_{R}(B)$ are finite and since a Jordan isomorphism is spectrum preserving it is easy to see that (ii) implies each of the assertions $(v)-(v i i)$.

Theorem 4.2. Let $\mathrm{A}$ be a unital $\mathrm{C}^{*}$-algebra of real rank zero and $\mathrm{B}$ a Banach algebra. Suppose that the linear map $\theta: \mathrm{A} \rightarrow \mathrm{B}$ is surjective up to the inessential ideal. Consider the following assertions. Then $(\mathfrak{i})-(v)$ are equivalent and $(v i)-(v i i i)$ imply $(v)$.

(i) $\theta$ is $\sigma_{e}$-preserving;

(ii) $\theta$ is $\partial \sigma_{e}$-preserving;

(iii) $\theta$ is $\eta \sigma_{e}$-preserving;

(iv) $\theta$ preserves Fredholm elements in both directions and $\theta(1)=1(\bmod \mathrm{I}(\mathrm{B}))$.

(v) $\theta(\mathrm{I}(\mathrm{A})) \subseteq \mathrm{I}(\mathrm{B})$ and the induced map $\widehat{\theta}: \mathrm{C}_{\mathrm{R}}(\mathrm{A}) \rightarrow \mathrm{C}_{\mathrm{R}}(\mathrm{B})$ is a continuous Jordan isomorphism;

(vi) $\theta$ is $\sigma_{l e}$-preserving;

(vii) $\theta$ is $\sigma_{\text {re-preserving; }}$

(viii) $\theta$ is $\left(\sigma_{\mathrm{le}} \cap \sigma_{\mathrm{re}}\right)$-preserving;

Proof. It is obvious that $(\mathfrak{i}) \Rightarrow(\mathfrak{i}),(\mathfrak{i}) \Rightarrow(\mathfrak{i i i}),(\mathfrak{i}) \Leftrightarrow(\mathfrak{i} v)$ and $(v)$ implies each of the statements (i) $-(\mathfrak{i v})$.

If each of the statements $(i)-(i i i)$ holds then $r_{e}(\theta(x))=r_{e}(x)$ and Lemma 3.1 implies that $\theta(I(A)) \subseteq I(B)$. Thus the induced map $\widehat{\theta}: C_{R}(A) \rightarrow C_{R}(B)$ is a spectral isometry and so is injective. By Theorem 3.6, $\widehat{\theta}$ is unital and preserves idempotents. Let $p$ and $q$ be orthogonal projections in $A$. Then, $p+q$ is a projection and

$$
\widehat{\theta}(p)+\widehat{\theta}(q)=(\widehat{\theta}(p)+\widehat{\theta}(q))^{2}=\widehat{\theta}(p)+\widehat{\theta}(p) \widehat{\theta}(q)+\widehat{\theta}(q) \widehat{\theta}(p)+\widehat{\theta}(q) .
$$


It follows that $\widehat{\theta}(p) \widehat{\theta}(q)+\widehat{\theta}(q) \widehat{\theta}(p)=0$ and hence $\widehat{\theta}(p) \widehat{\theta}(q)=\widehat{\theta}(p) \widehat{\theta}(q) \widehat{\theta}(p)=\widehat{\theta}(q) \widehat{\theta}(p)$. Therefore, $\widehat{\theta}(p)$ and $\widehat{\theta}(q)$ are orthogonal idempotents. Let $a=\sum_{j=1}^{n} \lambda_{j} p_{j}$ be a linear combination of mutually orthogonal projections $p_{1}, \ldots, p_{n} \in A$. Then,

$$
\widehat{\theta}\left(a^{2}\right)=\widehat{\theta}\left(\sum_{j=1}^{n} \lambda_{j}^{2} p_{j}\right)=\sum_{j=1}^{n} \lambda_{j}^{2} \widehat{\theta} p_{j}=(\widehat{\theta}(a))^{2}
$$

for $\widehat{\theta}\left(p_{1}\right), \ldots, \widehat{\theta}\left(p_{n}\right)$ are mutually orthogonal idempotents. Since every selfadjoint element in $A$ is the norm limit of finite linear combinations of mutually orthogonal projections and $\widehat{\theta}$ is continuous, $\widehat{\theta}\left(a^{2}\right)=(\widehat{\theta}(a))^{2}$ for every selfadjoint element $a$ in $A$. Replacing $a$ by $a+b$ in this identity yields $\widehat{\theta}(a b+b a)=\widehat{\theta}(a) \widehat{\theta}(b)+\widehat{\theta}(b) \widehat{\theta}(a)$ for all $a, b \in A_{s a}$. Suppose $a=a_{1}+i a_{2}$ with $a_{1}, a_{2} \in A_{s a}$. By the above argument

$$
\begin{aligned}
\widehat{\theta}\left(a^{2}\right) & =\widehat{\theta}\left(a_{1}^{2}+i\left(a_{1} a_{2}+a_{2} a_{1}\right)-a_{2}^{2}\right) \\
& =\widehat{\theta}\left(a_{1}\right)^{2}+i\left(\hat{\theta}\left(a_{1}\right) \widehat{\theta}\left(a_{2}\right)+\hat{\theta}\left(a_{2}\right) \hat{\theta}\left(a_{1}\right)\right)-\widehat{\theta}\left(a_{2}\right)^{2}=(\widehat{\theta}(a))^{2} .
\end{aligned}
$$

This proves that $\widehat{\theta}$ is a Jordan isomprphism and $(v)$ holds.

$(v i) \Rightarrow(v)$. Since $\partial \sigma_{e}(\theta(x)) \subseteq \sigma_{l e}(\theta(x))$ for all $x \in A$, we have

$$
\begin{aligned}
r_{e}(\theta(x)) & =\max \left\{|\lambda|: \lambda \in \sigma_{l e}(\theta(x))\right\} \\
& =\max \left\{|\lambda|: \lambda \in \sigma_{l e}(x)\right\}=r_{e}(x) .
\end{aligned}
$$

Lemma 3.1 implies that $\theta(I(A)) \subseteq I(B)$. The induced map $\widehat{\theta}: C_{R}(A) \rightarrow C_{R}(B)$ is $\sigma_{l}$-preserving and by Theorem 3.6, $\widehat{\theta}$ is a bijective unital linear map preserving idempotents and hence is a Jordan isomorphism. $(v i i) \Rightarrow(v)$ and $(v i i i) \Rightarrow(v)$ are proved similarly.

The following corollary is a direct consequence of Theorem 4.2 and [7, Corollary 5.3].

Corollary 4.3. Let $\mathrm{A}$ be a $\mathrm{C}^{*}$-algebra of real rank zero and $\mathrm{B}$ a $\mathrm{C}^{*}$-algebra. For a surjective up to inessential ideal linear map $\theta: A \rightarrow B$ such that $\theta(1)=1(\bmod \mathrm{I}(\mathrm{B}))$, the conditions $(\mathrm{i})-(v)$ in the above theorem are equivalent to the following conditions.

(i) There exist $\alpha, \beta>0$ such that $\alpha \gamma_{e}(a) \leq \gamma_{e}(\theta(a)) \leq \beta \gamma_{e}(a)$, for all $a \in A$.

(ii) $\sigma_{g e}(\theta(a))=\sigma_{g e}(a)$, for all $a \in A$.

Acknowledgement. The authors would like to thank the referee for valuable comments and suggestions.

Received: November 2012. Accepted: March 2013.

\section{References}

[1] B. Aupetit, A primer on spectral theory, Springer-Verlag, New York, 1991. 
[2] B. A. Barnes, G. J. Murphy, M. R. F. Smyth and T. T. West, Riesz and Fredholm theory in Banach algebras, Research Notes in Math. 67, Pitman, Boston, MA, 1982.

[3] M. Bendaoud and A. Bourhim, Essentially spectrally bounded linear maps, Proc. Amer. Math. Soc. 137 (2009) 3329-3334.

[4] M. Bendaoud, A. Bourhim, M. Burgos and M. Sarih, Linear maps preserving Fredholm and Atkinson elements of C*-algebras, Linear Multilinear Algebra, 57 (2009) 823-838.

[5] M. Bendaoud, A. Bourhim and M. Sarih, Linear maps preserving the essential spectral radius, Linear Alg. Appl. 428 (2008) 1041-1045.

[6] B. Blackadar, Operator Algebras: Theory of $C^{*}$-Algebras and von Neumann Algebras, Springer-Verlag, Berlin Heidelberg, 2006.

[7] A. Bourhim and M. Burgos, Linear maps preserving regularity in $\mathrm{C}^{*}$-algebras, Illinois $J$. Math. 53 (2009), 899-914.

[8] A. Bourhim, M. Burgos and V. Shulman, Linear maps preserving the minimum and reduced minimum moduli, J. Funct. Anal. 258 (2010) 50-66

[9] M-D. Choi, D. Hadwin, E. Nordgren, H. Radjavi and P. Rosenthal, On positive linear maps preserving invertibility, J. Funct. Anal. 59 (1984) 462-469

[10] J. L. Cui and J. C. Hou, Linear maps between Banach algebras compressing certain spectral functions, Rocky Mountain J. Math. 34 (2004) 565-585.

[11] S. O. Kim and C. Park, Linear maps on $\mathrm{C}^{*}$-algebras preserving the set of operators that are invertible in $\mathcal{A} / \mathcal{I}$. Canad. Math. Bull. 54 (1) (2011) 141-146.

[12] M. Mbekhta, Linear maps preserving the set of Fredholm operators, Proc. Amer. Math. Soc. 135 (2007) 3613-3619.

[13] M. Mbekhta, Linear maps preserving the minimum and surjectivity moduli of operators, Operators and Matrices 4 (4) (2010) 511-518.

[14] M. Mbekhta, L. Rodman, and P. Šemrl, Linear maps preserving generalized invertibility, Integr. Equ. Oper. Theory 55 (2006) 93-109.

[15] M. R. F. Smyth, Riesz theory in Banach algebras, Math. Z. 145 (1975) 145-155.

[16] M. R. F. Smyth, Fredholm theory in Banach algebras, Banach Center Publications, 8 (1982) 403-414. 